\title{
Imaging and histological appearance of pleomorphic hyalinizing angiectatic tumors: A case series and literature review
}

\author{
COUGER JIMENEZ JARAMILLO ${ }^{1}$, JOHN WOJCIK $^{2}$, KRISTY WEBER $^{3}$ and RONNIE SEBRO ${ }^{1}$ \\ Departments of ${ }^{1}$ Radiology, ${ }^{2}$ Pathology and ${ }^{3}$ Orthopedic Surgery, University of Pennsylvania, Philadelphia, PA 19104, USA
}

Received February 26, 2017; Accepted June 15, 2017

DOI: $10.3892 / 01.2018 .7929$

\begin{abstract}
Pleomorphic hyalinizing angiectatic tumors (PHATs) are rare mesenchymal soft tissue tumors of uncertain lineage and intermediate malignancy. The present study assesses two cases of PHAT and discusses the histological and immunophenotypical features, as well as the imaging appearance of these tumors on ultrasound, computed tomography (CT), magnetic resonance imaging and positron emission tomography/CT scans. The current study also reviews the literature and discusses the clinical management of these tumors. Wide local excision with tumor free margins is the current recommended treatment for PHAT. Surgical excision may be combined with low-dose radiation to reduce the risk of local recurrence. Patients should be followed up with serial imaging, as PHAT lesions tend to recur locally.
\end{abstract}

\section{Introduction}

Pleomorphic hyalinizing angiectatic tumors (PHATs) were first described by Smith et al (1) in 1996, and are rare mesenchymal tumors of uncertain lineage and intermediate malignancy (1-3). PHATs are defined by the presence of clusters of variably-sized thin-walled ectatic vessels that are infiltrated and surrounded by amorphous fibrin-rich hyaline material $(1,2)$. The angiectatic vessels characteristic of PHATs are surrounded by spindle-shaped, plump and round pleomorphic cells commonly arranged in sheets or, more rarely, in fascicles (1). Often, a subset of cells adjacent to the vessels contains intracytoplasmic hemosiderin. The pleomorphic cellular population is composed of large single

Correspondence to: Dr Ronnie Sebro, Department of Radiology, University of Pennsylvania, 3400 Spruce Street, Philadelphia, PA 19104, USA

E-mail: ronnie.sebro@uphs.upenn.edu

Abbreviations: PHAT, pleomorphic hyalinizing angiectatic tumors; CT, computed tomography; MRI, magnetic resonance imaging; PET/CT, positron emission tomography/computed tomography

Key words: pleomorphic hyalinizing angiectatic tumors, magnetic resonance imaging, computed tomography, ultrasound, positron emission tomography/computed tomography and multinucleate cells with abundant cytoplasm, enlarged irregular nuclei and, in certain cases, large intranuclear inclusions (1). Mitotic figures are rare (1). PHATs contain a mixed chronic inflammatory infiltrate that is notable for the presence of mast cells, but also includes lymphocytes, plasma cells and eosinophils $(1,3)$. The main mass of a PHAT may be accompanied by a partially myxoid spindle-cell component on the periphery, a potential precursor lesion to a classical PHAT that was described as an early PHAT by Folpe and Weiss in 2004 (2). The first cytogenetic PHAT data published revealed an unbalanced translocation of chromosomes 1 and 3 and chromosomes 1 and 10, with breakpoints mapped to transforming growth factor- $\beta$ receptor 3 (TGFBR3) and meningioma-expressed antigen 5 (MGEA5) (4). Subsequent fluorescence in situ hybridization and single nucleotide polymorphism analyses of PHATs demonstrated that some, but not all, are characterized by TGFBR3 and MGEA5 gene rearrangements (5-7).

There are few published reports describing the imaging appearance of PHATs. A PHAT typically appears as a soft tissue mass without osseous changes or calcification (8-10). In cases in which magnetic resonance imaging (MRI) is utilized, the PHAT presents as a soft-tissue mass that may be accompanied by edema or hematoma. The PHAT appears hypointense-isointense on T1-weighted sequences, heterogeneously isointense-hyperintense on T2-weighted sequences, and exhibits enhancement following administration of intravenous contrast $(4,8,11-18)$. The current study presents an assessment of two patients with similar PHATs, and describes the key imaging, histological and immunophenotypical findings of these tumors.

\section{Case reports}

Case A. A 50-year old male presented in 2016 to our institution with an 18-month history of an enlarging right buttock mass. The patient first noticed the mass following trauma and subjectively considered that the mass grew to be the size of a grapefruit. The patient denied experiencing any pain, except when sleeping on the right side; at the time of presentation, the patient was taking no pain medication and denied any radicular symptoms, night pain or weight loss. The patient also had normal muscle strength in the right lower extremity. A physical examination revealed a palpable, non-tender mobile mass in the right buttock. A contrast-enhanced computed 
tomography (CT) scan of the pelvis revealed an $8.0 \times 7.7 \times 8.6$ $\mathrm{cm}$ heterogeneously enhancing subcutaneous soft tissue mass overlying the right gluteus maximus (Fig. 1A). There was a prominent feeding artery noted as arising from the right profunda artery (Fig. 1B). MRI was performed using a Siemens Verio 3T MRI machine (Siemens AG, Munich, Germany). T1-weighted sequences (repetition time (TR)/echo time (TE), 700/24 ms; slice thickness, $3 \mathrm{~mm}$; interslice gap, $0.9 \mathrm{~mm}$; acquisition matrix, 448x336); short-tau inversion recovery (STIR) sequences (TR/TE, 3400/48 ms; slice thickness, 3 $\mathrm{mm}$; interslice gap, $0.9 \mathrm{~mm}$; and acquisition matrix, 256x192); and T1-weighted contrast-enhanced sequences with fat saturation (TR/TE, 638/23 ms; slice thickness, $4 \mathrm{~mm}$; interslice gap, $0.4 \mathrm{~mm}$; and acquisition matrix 320x256) were obtained. T1-weighted imaging revealed the presence of a heterogeneous predominantly isointense mass (Fig. 2A) with multiple areas of T1 signal hyperintensity (possibly representing areas of hemorrhage) and multiple areas of $\mathrm{T} 1$ signal hypointensity. The lesion was superficial to the right gluteus maximus fascia and present in the subcutaneous tissues (Fig. 2B). Multiple flow voids consistent with small arteries were observed in the lesion (Fig. 2C). There was no lymphadenopathy and no osseous involvement. The mass exhibited heterogeneous enhancement following administration of intravenous contrast (Fig. 2D). The lesion was heterogeneously hypoechoic with internal globular and punctate hyperechoic foci when evaluated by ultrasound (Fig. 3).

Histologic sections $5 \mu \mathrm{m}$ thickness were deparaffinized and stained at room temperature with hematoxylin $(5 \mathrm{~min})$ and eosin $(40 \mathrm{sec})$ on a Tissue Tek Prisma Automatic Stainer (Sakura Finetek USA, Inc., Torrance, CA, USA). On examination with light microscopy (variable objectives, magnification range $25-400 x$ ), the sections revealed a spindle cell lesion with sparsely sampled, hyalinized vessels. The cellular component was largely bland and spindled, with intermixed hemosiderin-laden macrophages and scattered large, atypical cells. Immunohistochemistry on formalin-fixed, paraffin-embedded tissue was performed using a Leica Bond-IIITM instrument using the Bond Polymer Refine Detection System according to the manufacturer's protocols (DS9800; Leica Microsystems, Inc., Buffalo Grove, IL, USA). Heat-induced epitope retrieval was performed for $20 \mathrm{~min}$ with ER1 or ER2 solution (Leica Biosystems) according to the manufacturer's protocols. The tissues were then incubated with the following primary antibodies at room temperature for $15 \mathrm{~min}$ : CD34 (ready to use dilution; cat no. PA0354; Leica Biosystems GmbH, Wetzlar, Germany), pancytokeratin (dilution 1:75; cat no. MU357-UC; BioGenex Laboratories, Inc., San Ramon, CA, USA), S100 (ready to use dilution; cat no. IR50461; Dako; Agilent Technologies, Inc., Santa Clara, CA, USA), smooth muscle actin (dilution, 1:600; cat no. M0851; Dako), epithelial membrane antigen (dilution, 1:100, cat no. M0613, Dako) and collagen IV (dilution 1:100; cat no. M0785; Dako). All interpretation of immunohistochemical stains was conducted with routine light-microscopy (variable magnification, x25-400). On initial biopsies, immunohistochemical studies were inconclusive with respect to line of differentiation. No high-grade features were seen. Given the small sample size and the lack of definitive histological features, the non-specific diagnosis of 'spindle-cell neoplasm' was determined, with a differential diagnosis that included PHAT. Positron-emission tomography/ computed tomography (PET/CT) demonstrated a mildly hypermetabolic lesion (maximum standardized uptake value of 4.2) with no evidence of hypermetabolic metastases (Fig. 4). The lesion was resected with wide surgical margins. The excised specimen contained a circumscribed tumor with prominent thin-walled, hyalinized vessels (Fig. 5). There were areas of hemorrhage and vascular thrombosis. An intermediate density of bland ovoid cells with rounded nuclear contour and occasional small nucleoli, along with admixed hemosiderin-laden cells and scattered large single- and multi-nucleated atypical cells was also observed. Mitotic activity was low $(<1 / 10$ mitoses per high-power field). On the basis of these morphological findings, the patient was diagnosed with PHAT.

Case B. A 72-year-old male presented to our institution in 2013 with a mass along the right buttock that had been slowly growing for almost 30 years. The mass was mildly uncomfortable when the patient sat. The patient also reported no unintentional weight loss or lymphadenopathy. A physical examination revealed a palpable, mobile, non-tender mass measuring approximately $10 \mathrm{~cm}$ in size. MRI was performed using a Siemens Espree 3T MRI (Siemens AG). T2-weighted sequences (TR/TE, 6,721/167 ms; slice thickness, $5 \mathrm{~mm}$; interslice gap, $1 \mathrm{~mm}$; and acquisition matrix, 320x320) and T1-weighted gradient imaging with fat saturation following administration of intravenous contrast (TR/TE, 170/2.37 ms; slice thickness, $5 \mathrm{~mm}$; interslice gap, $1 \mathrm{~mm}$; and acquisition matrix, 256x 218) were obtained. The lesion was superficial to the fascia of the right gluteus maximus and measured $\sim 6.3 \times 10.4 \times 10.2 \mathrm{~cm}$. The lesion exhibited a heterogeneously hyperintense T2 intensity signal (Fig. 6A) and heterogeneous enhancement following administration of intravenous contrast; there were two prominent arterial vessels originating from the right internal iliac artery supplying the lesion (Fig. 6B and C). The patient underwent gross total resection of the tumor with negative margins. The histological findings were similar to those in case A, although this patient had reduced cellularity and more prominent areas of myxoid stroma (Fig. 7). As in case A, the tumor was composed of a mixture of bland spindle cells with occasional large irregular cells that had retained cytoplasm and large atypical nuclei. There was no discernable mitotic activity or necrosis (Fig. 7). In the immunohistochemical studies, the spindled tumor cells were positive for cluster of differentiation CD34 expression, focally positive for pancytokeratin expression, and negative for the expression of S100, smooth muscle actin, epithelial membrane antigen and collagen IV. The final histological diagnosis was a PHAT.

The requirement for patient consent for publication of the study was waived and ethical approval was provided by the Institutional Review Board of the University of Pennsylvania (Philadelphia, PA, USA).

\section{Discussion}

To date, $<100$ cases of PHAT have been reported worldwide $(14,19)$. The World Health Organization currently classifies PHAT as a benign neoplasm of uncertain differentiation (3). No reports of metastatic PHATs exist; however, it has 

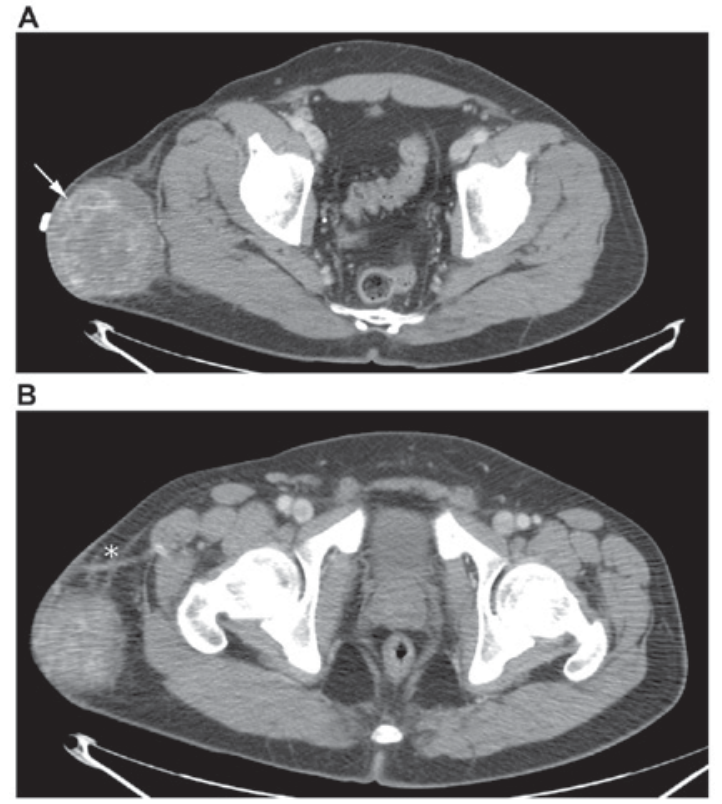

Figure 1. (A) Axial contrast-enhanced CT image of the pelvis indicating a heterogeneously enhancing mass in the subcutaneous tissues superficial to the right gluteus maximus (white arrow) from case A. (B) Axial contrast-enhanced CT image of the pelvis indicating a prominent artery (white asterisk) arising from the right profunda artery and supplying the heterogeneously enhancing mass in the subcutaneous tissues superficial to the right gluteus maximus. CT, computed tomography.l
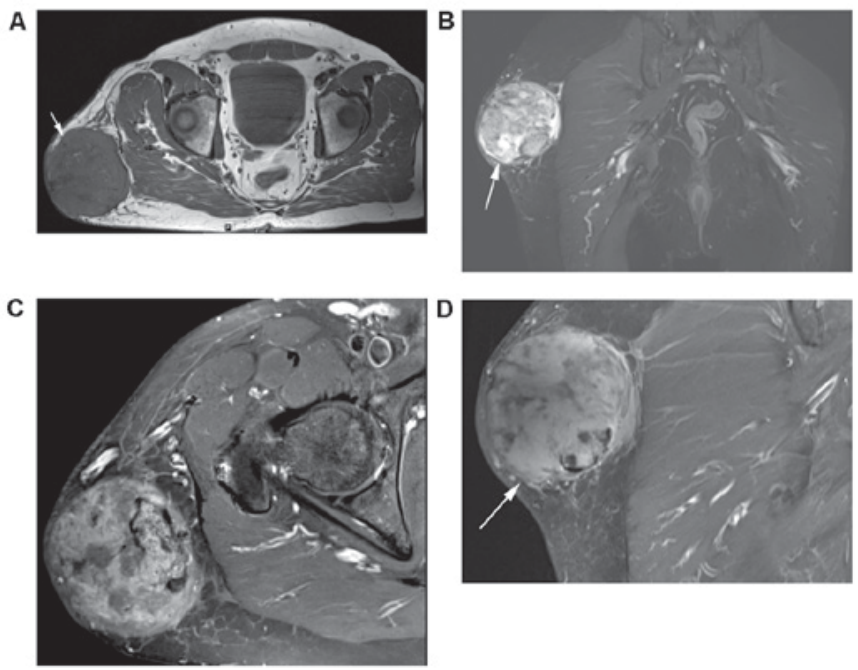

Figure 2. (A) Axial T1-weighted MR image of the pelvis depicting a T1-heterogeneous mass (white arrow) with areas of internal T1-hyperintensity and T1-hypointensity from the patient (case A). (B) Coronal STIR MR image of the pelvis indicating a heterogeneously $\mathrm{T} 2$-hyperintense subcutaneous mass (white arrow) along the right gluteus maximus fascia. (C) Axial STIR MR image of the pelvis indicating multiple flow voids within the mass consistent with small vessels. (D) Coronal T1-weighted MR image of the pelvis following administration of intravenous contrast with fat saturation indicating a heterogeneously enhancing mass along the fascia of the right gluteus maximus (white arrow). MR, magnetic resonance; STIR, short tau inversion recovery sequence.

been known to recur as sarcoma in rare cases $(2,20)$. Owing to its locally aggressive behavior and high recurrence rate (33-50\%), it has been argued that a PHAT should be classified as a mesenchymal tumor of intermediate malignancy (2).

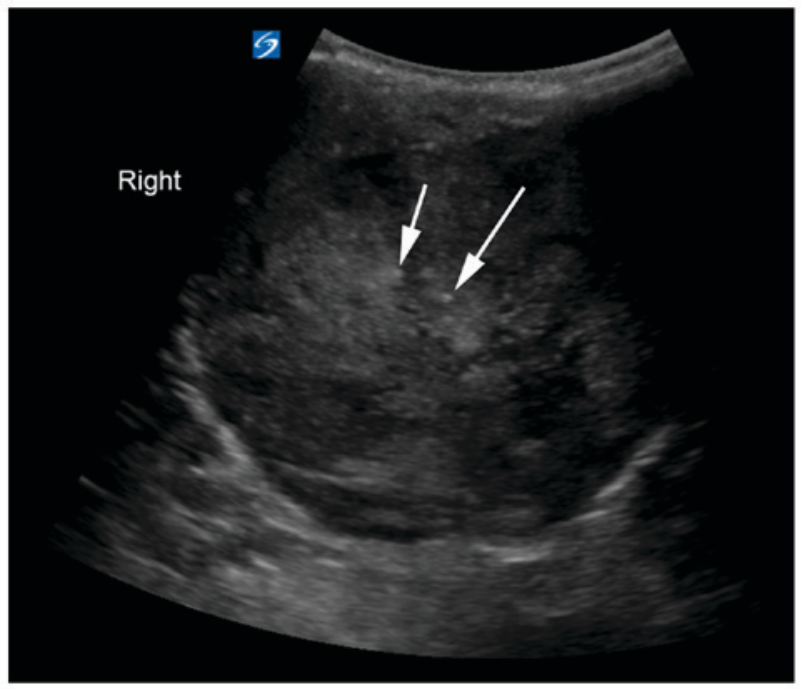

Figure 3. Transverse sonographic image $(5 \mathrm{MHz})$ of the right buttock from the patient (case A) depicting a heterogeneously hypoechoic mass with hyperechoic rim and internal globular and punctate hyperechoic foci (white arrows).

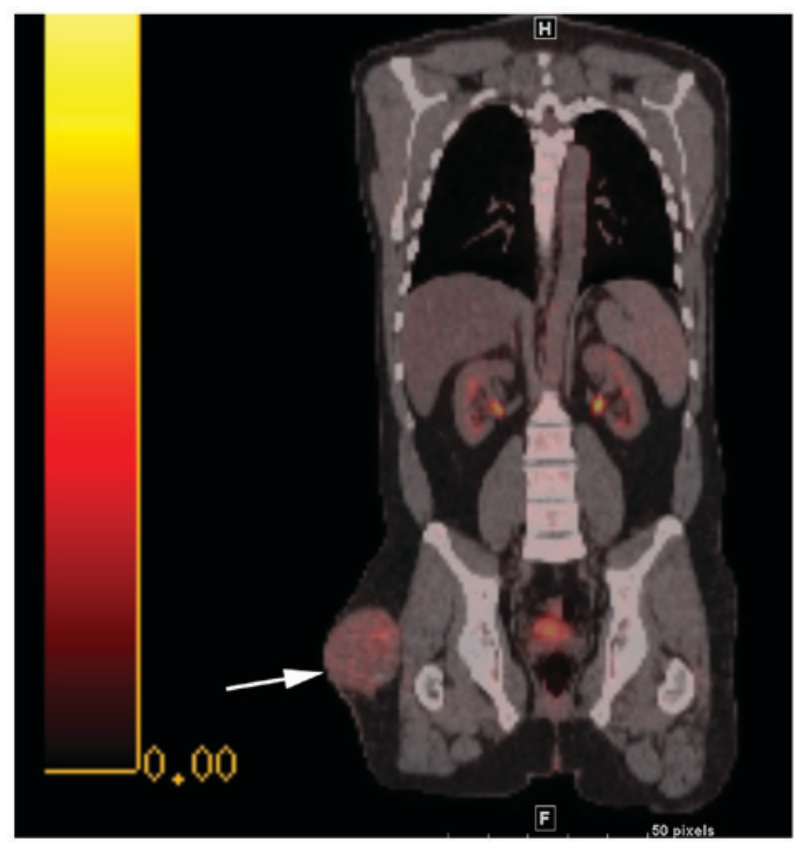

Figure 4. Coronal fused positron emission tomography/computed tomography image of the patient (case A)indicating a mildly FDG-avid mass along the right buttock (white arrow). No other foci of abnormal increased FDG activity were noted. FDG, ${ }^{18} \mathrm{~F}$-fludeoxyglucose.

PHAT was first described by Smith et al (1) in 1996. The etiology of PHAT is not well understood and a number of competing theories persist regarding the formation of classic PHAT (1-3). The original description of the tumor by Smith et al (1) posited that the encroachment of a developing PHAT onto the vessels of healthy tissue leads to the release of the plasma contents of damaged endothelial cells, and precipitates a process of organization that results in the tumor's characteristic perivascular hyalinization (1). Smith et al (1) further proposed that the release of vasoactive mast cell products in response to tissue injury from a developing PHAT 
A

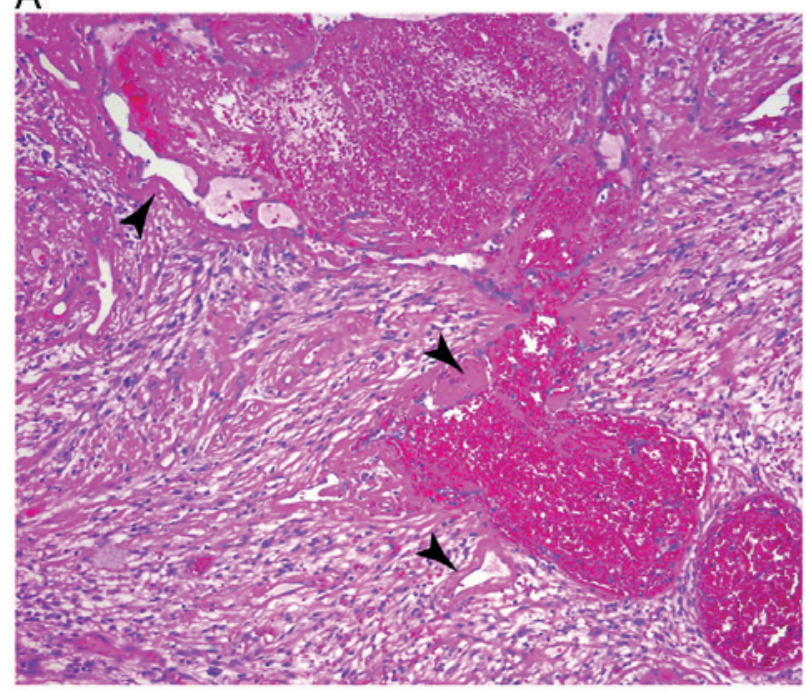

B

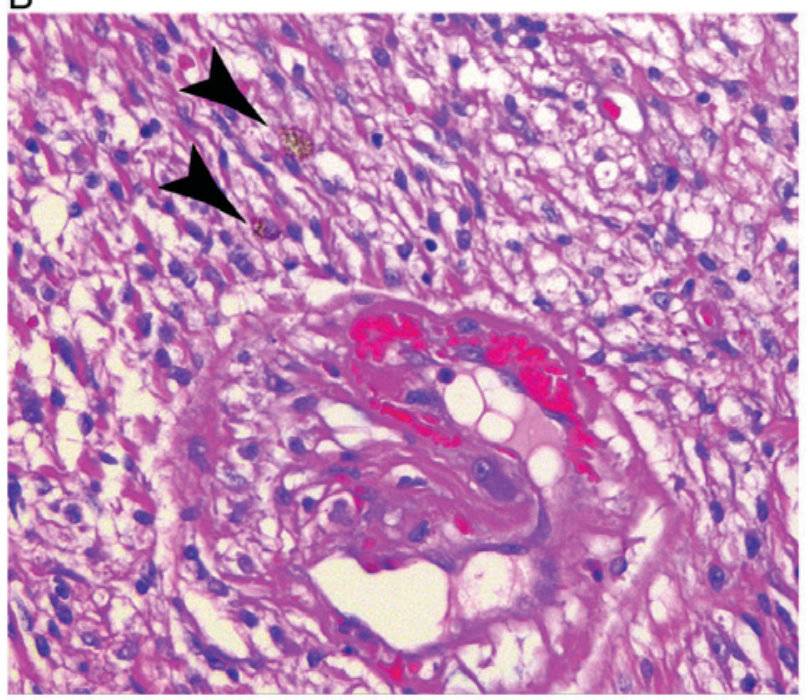

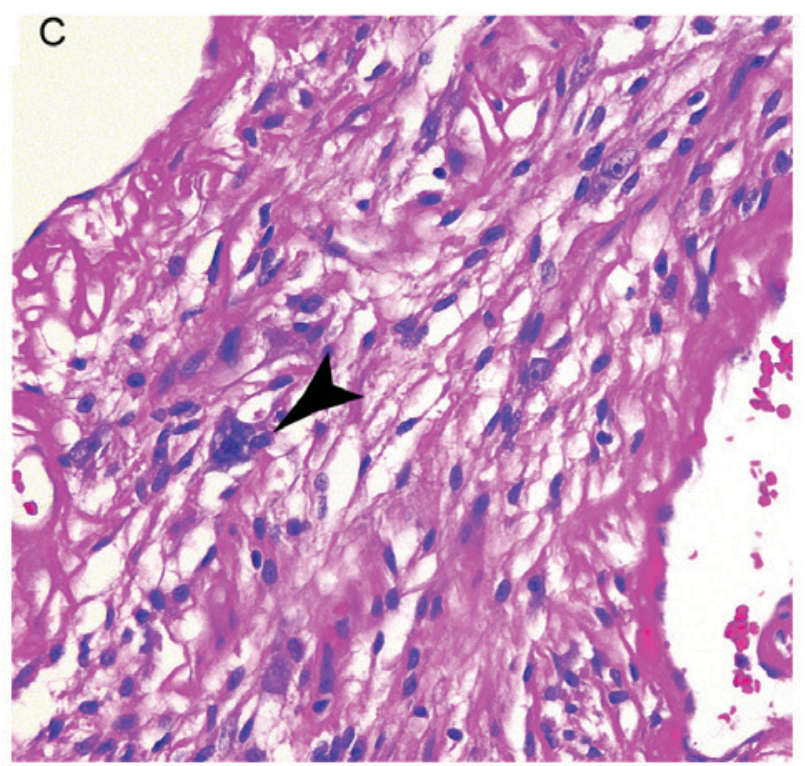

Figure 5. Histological findings in case A, hematoxylin and eosin. (A) At an intermediate magnification (x100), the tumor consists of a spindle cell tumor with large, thin walled vessels and nascent thrombus formation. The vessel walls are surrounded by prominent hyaline material (arrowheads). There are areas of hemorrhage and a mild, chronic inflammatory infiltration and scattered atypical cells. (B) At a higher magnification (x200), hemosiderin-laden mononuclear phagocytes (arrowheads) and (C) large atypical cells (arrowhead) are apparent.
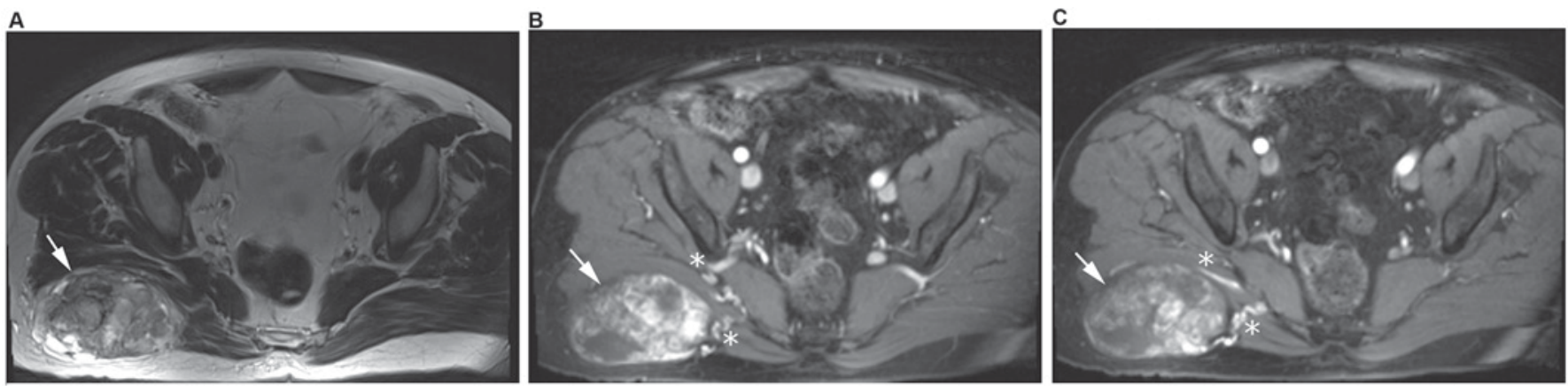

Figure 6. (A) Axial T1-weighted MR image of the pelvis without fat saturation depicting a heterogeneously T1 hyperintense lesion (white arrow) with mass effect on the right gluteus maximus from the patient (case B). There are areas of internal increased T1 signal suggestive of fat or hemorrhage. (B) Axial T1-weighted MR image of the pelvis with fat saturation following administration of intravenous contrast depicting a heterogeneously enhancing mass along the right gluteus maximus without invasion of the gluteus maximus (white arrow) and two prominent feeding arterial vessels (white asterisks). (C) Axial T1-weighted MR image of the pelvis with fat saturation following administration of intravenous contrast depicting a heterogeneously enhancing mass along the right gluteus maximus without invasion of the gluteus maximus (white arrow) and two prominent feeding arterial vessels (white asterisks). MR, magnetic resonance. 

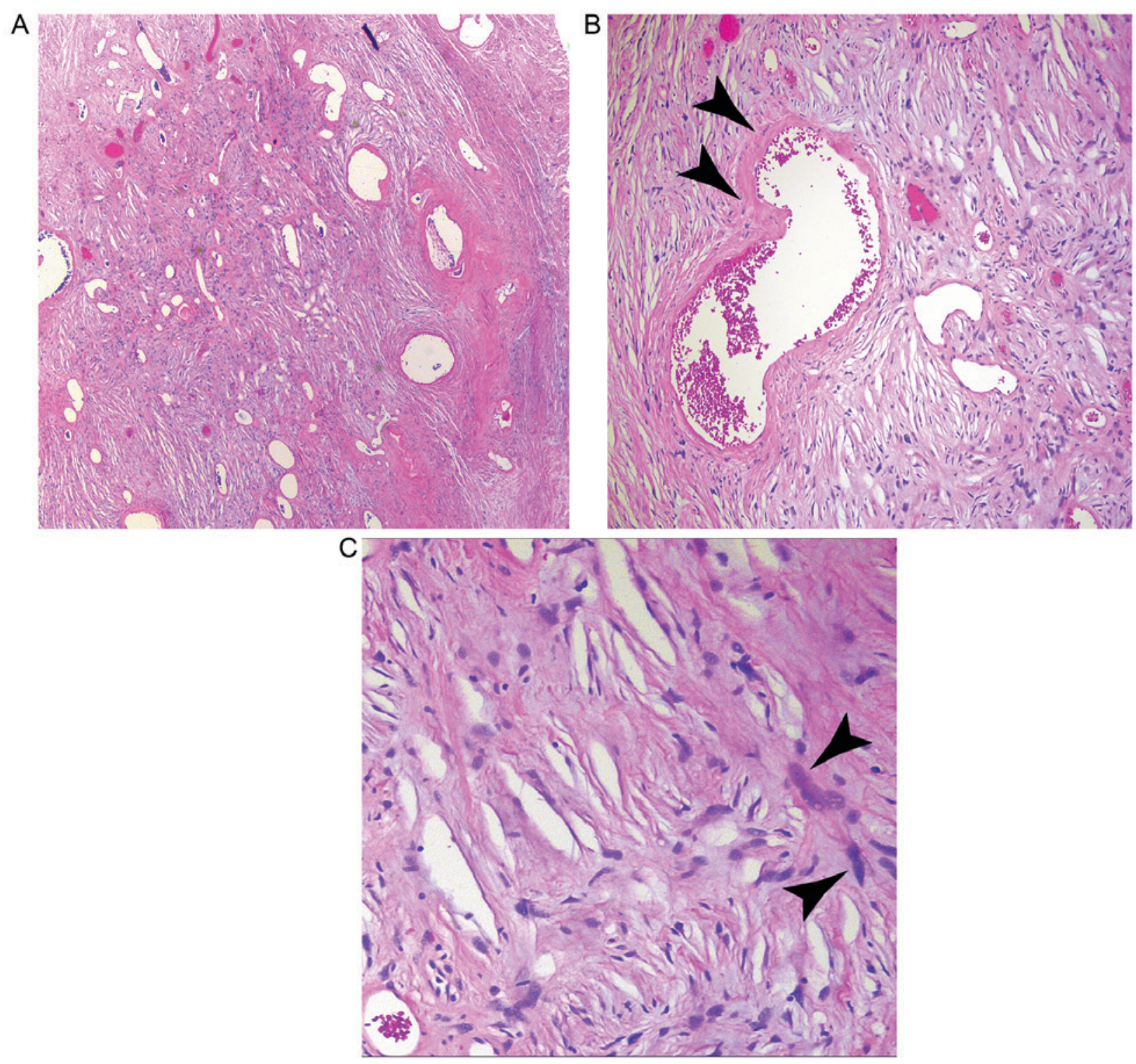

Figure 7. Histological findings in case B, hematoxylin and eosin. (A) At a low magnification (x50), the tumor is similar in appearance to case A, although with more variable cellularity and a more pronounced gray-blue myxoid stroma. (B) The thin, ectatic vessels contain perivascular hyaline material (arrowheads; magnification, x100). There are admixed large, atypical cells visible at low magnification and (C) highlighted at higher magnification (arrowheads; magnification, $\mathrm{x} 200)$.

may be a cause of increased vessel permeability during development (1). Alternately, Folpe and Weiss (2) argue that early PHAT, characterized by low-medium spindle cell density, rare pleomorphic cells and myxoid extracellular matrix, is a precursor lesion to classic PHAT (2). PHAT typically presents in the subcutaneous tissues of the lower extremities, but may also develop intramuscularly, and has been documented in the arm, axilla, back, buccal mucosa, buttock, chest, hand, perineum, popliteal fossa and retroperitoneum $(2,3,13,21)$. The most common locations of PHATs, based on the published literature, include the leg $(18 / 97 ; 18.6 \%)$, thigh $(17 / 97 ; 17.5 \%)$, foot $(14 / 97 ; 14.4 \%)$ and the ankle $(10 / 97 ; 10.3 \%)(1-41)$. PHAT typically presents in adults; the cases presented in the published literature (97 patients) range in age from 10-89 years, with a median age of 57. PHAT demonstrates a slight predilection for females over males; the literature reviewed in the present study demonstrates a male to female ratio of 3:4 out of 97 reported cases $(2,22,23)$. No information is currently available concerning race and, as $<10 \%$ of reported cases include this metric, no claims can be made regarding a predilection. Table I presents a list of all previously published cases of PHAT, including data on age, sex, race, tumor size, location and treatment $(1,2,4,5,8-41)$.

PHAT often rises to medical attention following several years of slow growth (1-3). Upon clinical examination, PHAT typically presents grossly as an oval-round lobulated mass that varies in color from tan to maroon, and which may be associated with bruising or surrounding hematoma $(2,9,10)$. PHAT may present as partially or predominantly cystic $(1,12)$, may be painless, painful or tender upon examination, and may be accompanied by necrosis in rare cases $(1,14)$.

The imaging appearance of PHAT is non-specific. Typical evaluation of any soft-tissue mass includes radiography of the region, followed by contrast-enhanced MRI evaluation 


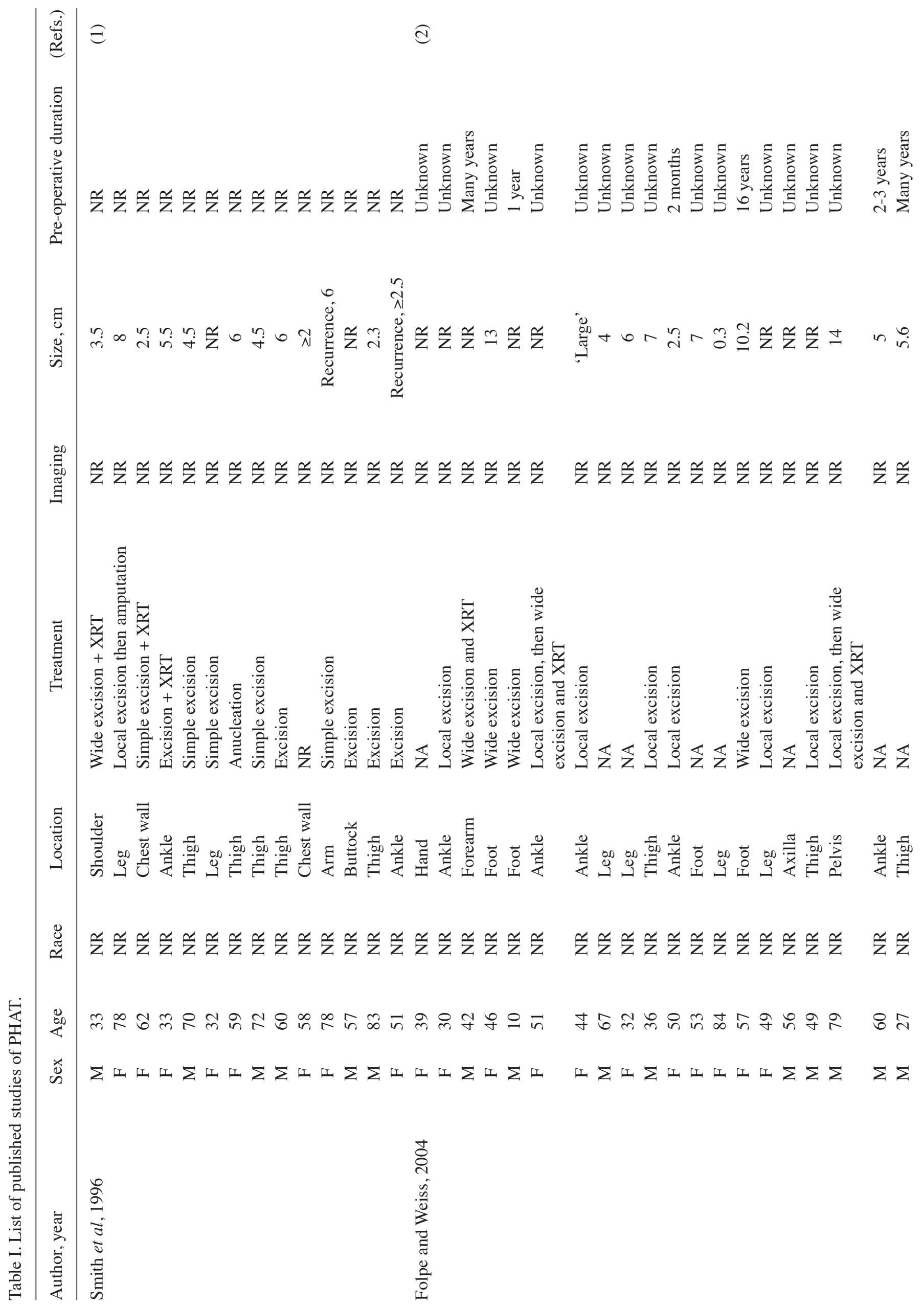




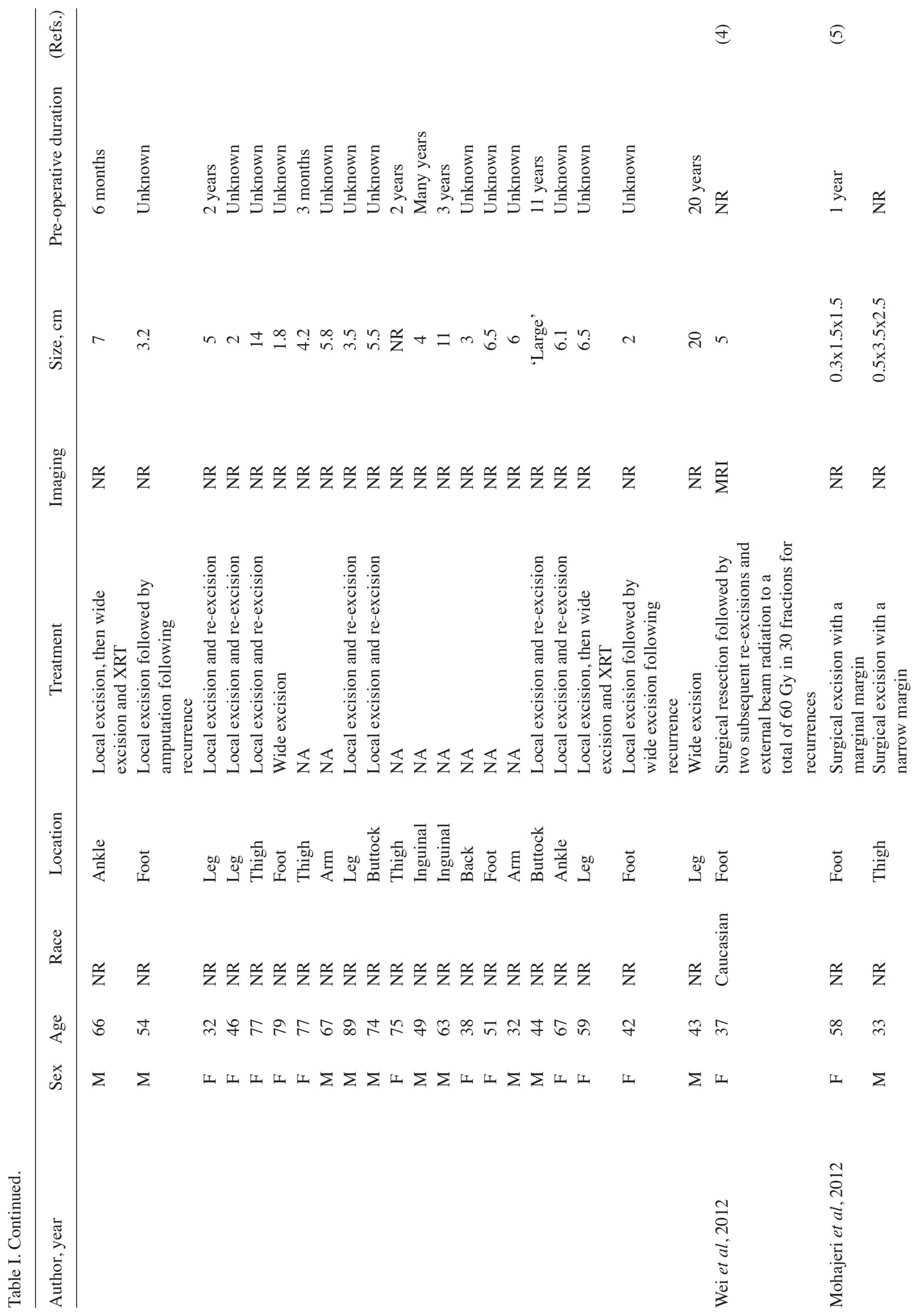




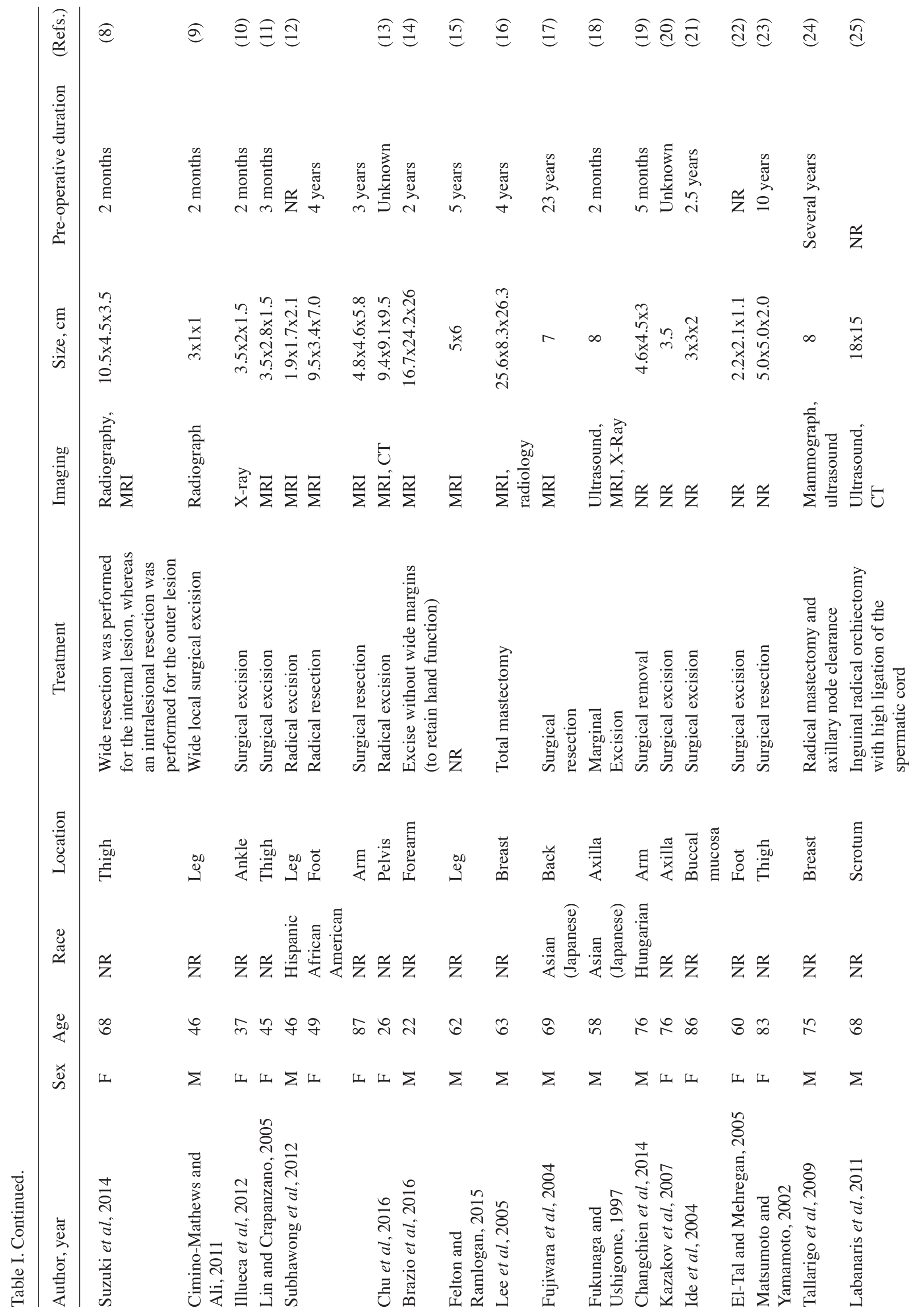




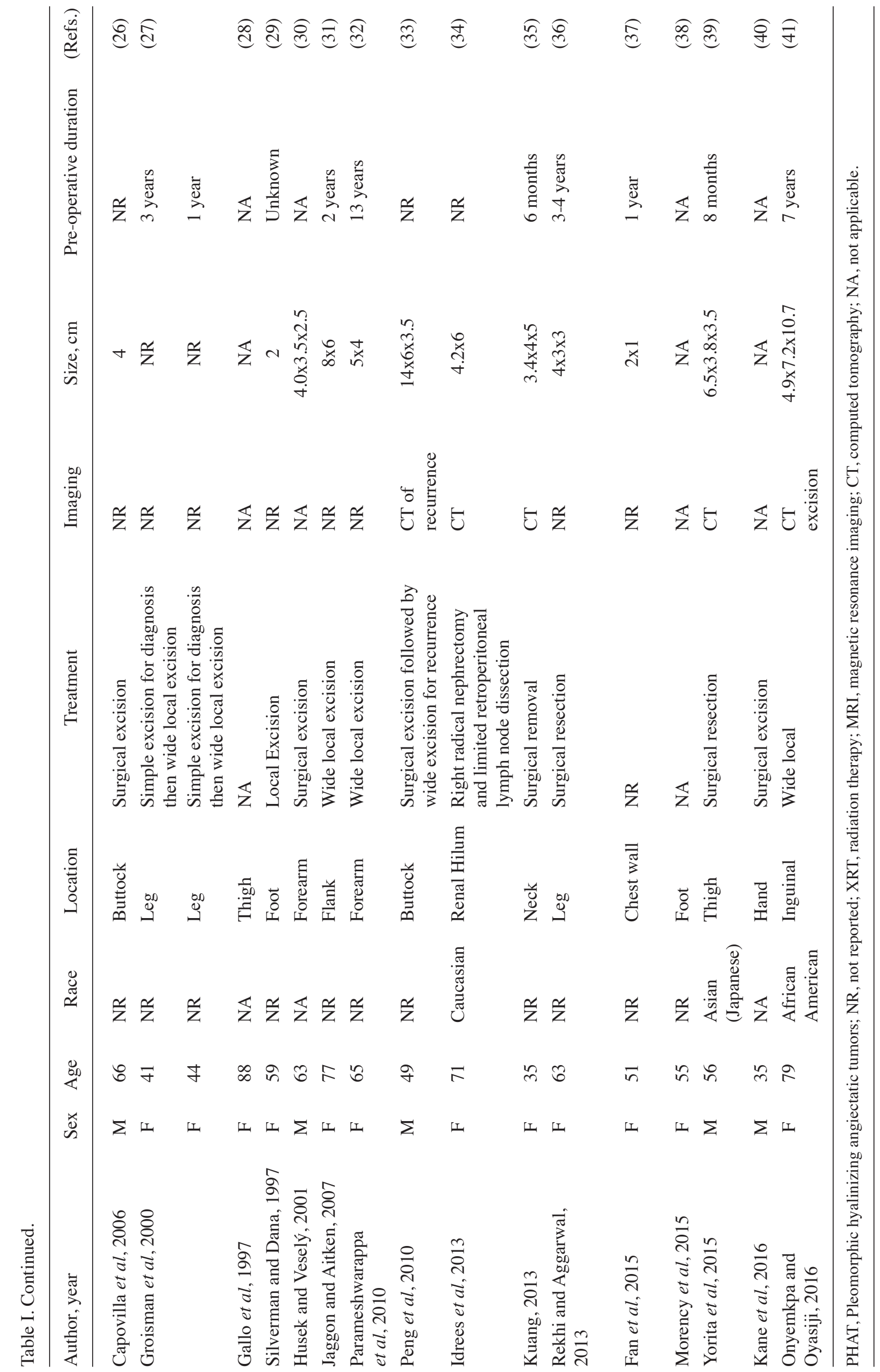


and then by biopsy if clinically warranted. Limited imaging studies of PHAT exist in the literature and the majority of data are in the form of single case reports. In cases in which radiographs are published, there is consensus that PHAT typically appears as a soft tissue mass without osseous changes or calcifications; however, a single case with calcifications has been reported $(8-10,14,24)$. To the best of our knowledge, only three examples of the ultrasound appearance of PHAT have been described in the literature $(18,24,25)$. All three describe PHAT as a hypoechoic lesion with an internal hyperechogenic appearance, characterized by hyperechoic tissue interrupted by hypoechoic bands, which are presumably blood vessels $(18,24,25)$. In reports of PHAT using MRI, the tumor presents as a soft tissue mass that may be accompanied by edema or hematoma (8,11-15). PHAT appears hypointense to isointense with $\mathrm{T} 1$ weighting, heterogeneously hyperintense to isointense with T2 weighting, and exhibits heterogeneous enhancement following the intravenous administration of gadolinium-based contrast $(4,8,11-13,16-18)$. PHATs with well-demarcated and infiltrative borders have previously been described in the literature $(9,12)$. The two cases presented in the current study exhibited prominent borderline ectatic arterial vessels supplying the tumors and well-demarcated tumor borders. The imaging differential diagnosis includes subcutaneous metastasis, melanoma or a soft tissue sarcoma.

PHAT is notable for the presence of clusters of ectatic vessels surrounded by fibrin-rich hyaline material and cuffed by lamellae of collagen that extend into the mass of the tumor and trap individual cells, potentially forming pockets of hyalinized material $(1,2)$. PHATs contain spindle-shaped, plump and round pleomorphic cells $(1,2)$. Cells exhibit hyperchromasia, nuclear pleiomorphism and a lack of cytoplasmic differentiation; they may also contain hemosiderin, nuclear inclusions and cytoplasmic vimentin filaments $(9,26)$. Immunohistochemical analysis of PHATs typically reveal the presence of CD34 and may also detect expression of vimentin, B-cell lymphoma 2, factor XIIIa, vascular endothelial growth factor and CD99; PHAT lacks expression of S-100 protein, CD31, von Willebrand Factor, smooth muscle actin, desmin and epithelial membrane protein $(1,2,11,22,27)$. PHAT cells stained for Ki-67 rarely exhibit positive staining, consistent with the histological observation of few mitotically active cells $(1,22)$. Histological differential diagnoses include neurilemmoma, undifferentiated pleomorphic sarcoma (UPS), giant-cell angiofibroma and solitary fibrous tumor (SFT) $(1,3,11,22,23)$. PHAT can be distinguished from neurilemmoma by the absence of S-100, lack of encapsulation, and the absence of Antoni A and B areas $(11,22)$. PHAT can be distinguished from UPS by its infrequent mitotic figures and the presence of nuclear inclusions $(1,9,22)$. PHAT can be distinguished from by the presence of clusters of thin-walled vessels, atypical cells with bland cytoplasm and intranuclear inclusions (11).

Wide local excision with tumor free margins is the recommended treatment for PHAT $(1,2,16)$ as they may occur as a collision tumor with sarcomas such as myxofibrosarcomas (20). Surgical excision may be combined with low dose radiation to reduce the risk of local recurrence $(1,2,4)$. The patients were followed with serial imaging, as these lesions have a tendency to locally recur. One patient was followed lost to follow up after one year, and the other remains under active imaging surveillance (follow up of 1 year).

The present study reported and discusses the ultrasound, CT, MRI and PET/CT imaging appearance and correlating histology of two histologically verified cases of PHAT. Biopsy remains a requirement because imaging features are non-specific.

\section{Competing interests}

The authors declare that they have no competing interests.

\section{References}

1. Smith ME, Fisher C and Weiss SW: Pleomorphic hyalinizing angiectatic tumor of soft parts. A low-grade neoplasm resembling neurilemoma. Am J Surg Pathol 20: 21-29, 1996.

2. Folpe AL and Weiss SW: Pleomorphic hyalinizing angiectatic tumor: Analysis of 41 cases supporting evolution from a distinctive precursor lesion. Am J Surg Pathol 28: 1417-1425, 2004.

3. Fletcher CDM, World Health Organization and International Agency for Research on Cancer: WHO Classification of Tumours of Soft Tissue and Bone. 4th edition. IARC Press, Lyon, 2013.

4. Wei S, Pan Z, Siegal GP, Winokur TS, Carroll AJ and Jhala D: Complex analysis of a recurrent pleomorphic hyalinizing angiectatic tumor of soft parts. Hum Pathol 43: 121-126, 2012.

5. Mohajeri A, Kindblom LG, Sumathi VP, Brosjö O, Magnusson L, Nilsson J, Nord KH and Mertens F: SNP array and FISH findings in two pleomorphic hyalinizing angiectatic tumors. Cancer Genet 205: 673-676, 2012.

6. Antonescu CR, Zhang L, Nielsen GP, Rosenberg AE, Dal Cin P and Fletcher CD: Consistent $t(1 ; 10)$ with rearrangements of TGFBR3 and MGEA5 in both myxoinflammatory fibroblastic sarcoma and hemosiderotic fibrolipomatous tumor. Genes Chromosomes Cancer 50: 757-764, 2011.

7. Carter JM, Sukov WR, Montgomery E, Goldblum JR, Billings SD, Fritchie KJ and Folpe AL: TGFBR3 and MGEA5 rearrangements in pleomorphic hyalinizing angiectatic tumors and the spectrum of related neoplasms. Am J Surg Pathol 38: 1182-1192, 2014.

8. Suzuki K, Yasuda T, Hori T, Oya T, Watanabe K, Kanamori M and Kimura T: Pleomorphic hyalinizing angiectatic tumor arising in the thigh: A case report. Oncol Lett 7: 1249-1252, 2014.

9. Cimino-Mathews A and Ali SZ: Pleomorphic hyalinizing angiectatic tumor: Cytomorphologic features on fine-needle aspiration. Diagn Cytopathol 39: 214-217, 2011.

10. Illueca C, Machado I, Cruz J, Almenar S, Noguera R, Navarro S and Llombart-Bosch A: Pleomorphic hyalinizing angiectatic tumor: A report of 3 new cases, 1 with sarcomatous myxofibrosarcoma component and another with unreported soft tissue palpebral location. Appl Immunohistochem Mol Morphol 20: 96-101, 2012.

11. Lin O and Crapanzano JP: Fine-needle aspiration cytology of pleomorphic hyalinized angiectatic tumor: A case report. Diagn Cytopathol 32: 238-242, 2005.

12. Subhawong TK, Subhawong AP, Montgomery EA and Fayad LM: Pleomorphic hyalinizing angiectatic tumor: Imaging findings. Skeletal Radiol 41: 1621-1626, 2012.

13. Chu ZG, Liu MQ, Zhu ZY, Lv FJ and Ouyang Y: Pelvic retroperitoneal pleomorphic hyalinizing angiectatic tumor (PHAT) of soft tissue: A case report. BMC Med Imaging 16: 28, 2016.

14. Brazio PS, Morrison AL, Oh M, Goldberg N and Boutros CN: Large pleomorphic hyalinizing angiectatic tumor of the forearm: A multidisciplinary perspective. Surgery 159: 1471-1473, 2016.

15. Felton SJ and Ramlogan D: Spontaneous bruising overlying a subcutaneous swelling: Pleomorphic hyalinizing angiectatic tumor, a relatively new entity of intermediate malignancy. Int J Dermatol 54: 204-206, 2015.

16. Lee JC, Jiang XY, Karpinski RH and Moore ED: Pleomorphic hyalinizing angiectatic tumor of soft parts. Surgery 137: 119-121, 2005.

17. Fujiwara M, Yuba Y, Wada A, Ozawa T and Tanaka T: Pleomorphic hyalinizing angiectatic tumor of soft parts: Report of a case and review of the literature. J Dermatol 31: 419-423, 2004.

18. Fukunaga $\mathrm{M}$ and Ushigome S: Pleomorphic hyalinizing angiectatic tumor of soft parts. Pathol Int 47: 784-788, 1997. 
19. Changchien YC, Bocskai P, Kovács I, Hargitai Z, Kollár S and Török M: Pleomorphic hyalinizing angiectatic tumor of soft parts: Case report with unusual ganglion-like cells and review of the literature. Pathol Res Pract 210: 1146-1151, 2014.

20. Kazakov DV, Pavlovsky M, Mukensnabl P and Michal M: Pleomorphic hyalinizing angiectatic tumor with a sarcomatous component recurring as high-grade myxofibrosarcoma. Pathol Int 57: 281-284, 2007.

21. Ide F, Shimoyama $\mathrm{T}$ and Horie N: Pleomorphic hyalinizing angiectactic tumor of the buccal mucosa. J Oral Pathol Med 33: 451-453, 2004.

22. El-Tal AE and Mehregan D: Pleomorphic hyalinizing angiectatic tumor of soft parts: Case report and literature review. J Cutan Pathol 33: 361-364, 2006.

23. Matsumoto $\mathrm{K}$ and Yamamoto $\mathrm{T}$ : Pleomorphic hyalinizing angiectatic tumor of soft parts: A case report and literature review. Pathol Int 52: 664-668, 2002.

24. Tallarigo F, Squillaci S, Putrino I, Zizzi N and Bisceglia M Pleomorphic hyalinizing angiectatic tumor of the male breast: A heretofore unreported occurrence. Pathol Res Pract 205: 69-73, 2009.

25. Labanaris AP, Zugor V, Witt JH and Kuhn R: Pleomorphic hyalinizing angiectatic tumor of the scrotum. Urol J 8: 248-250, 2011.

26. Capovilla M, Birembaut P, Cucherousset J, Ploton D, de Saint-Maur PP, Fléjou JF and Lesec G: Pleomorphic hyalinizing angiectatic tumor of soft parts: Ultrastructural analysis of a case with original features. Ultrastruct Pathol 30: 59-64, 2006.

27. Groisman GM, Bejar J, Amar M and Ben-Izhak O: Pleomorphic hyalinizing angiectatic tumor of soft parts: Immunohistochemical study including the expression of vascular endothelial growth factor. Arch Pathol Lab Med 124: 423-426, 2000.

28. Gallo C, Murer B and Roncaroli F: Pleomorphic hyalinizing angiectasic soft-tissue tumor. Description of a case. Pathologica 89 531-535, 1997.

29. Silverman JS and Dana MM: Pleomorphic hyalinizing angiectatic tumor of soft parts: Immunohistochemical case study shows cellular composition by CD34+ fibroblasts and factor XIIIa+ dendrophages. J Cutan Pathol 24: 377-383, 1997.

30. Husek K and Veselý K: Pleomorphic hyalinizing angiectatic tumor. Cesk Patol 37: 177-181, 2001
31. Jaggon JR and Aitken RD: Pleomorphic hyalinizing angiectatic tumour of soft parts: A case report and review of the literature. West Indian Med J 56: 544-546, 2007.

32. Parameshwarappa S, Rodrigues G, Nagpal N and Rao L: Pleomorphic hyalinizing angiectatic tumor of soft parts involving the upper limb. Indian J Surg 72: 263-264, 2010.

33. Peng HC, Huang MT, Chen DJ, Leung TK and Chu JS: Pleomorphic hyalinizing angiectatic tumor of soft parts. J Formos Med Assoc 109: 616-620, 2010.

34. Idrees MT, Kieffer T and Badve S: Pleomorphic hyalinizing angiectatic tumor of renal hilum. Ann Diagn Pathol 16: 489-493, 2012.

35. Kuang P: CT findings of pleomorphic hyalinizing angiectatic tumor (PHAT) of soft parts of the neck. Jpn J Radiol 31: 204-207, 2013.

36. Rekhi B and Aggarwal S: Pleomorphic hyalinizing angiectatic tumor exhibiting intricate branching vasculature: An interesting pattern in a rare tumor. Indian J Pathol Microbiol 56: 321-323, 2013.

37. Fan C, Lin X, Yang L and Wang E: A subcutaneous pleomorphic hyalinizing angiectatic tumor of soft parts of the right chest wall: Report of a rare case. Int J Clin Exp Pathol 8: 11804-11808, 2015.

38. Morency E, Laskin W and Lin X: Cytologic and histologic features of pleomorphic undifferentiated sarcoma arising in a hybrid hemosiderotic fibrolipomatous tumor and pleomorphic hyalinizing angiectatic tumor: Report of an unusual case with a literature review. Acta Cytol 59: 493-497, 2015.

39. Yorita K, Ishihara A, Tokumitsu T, Minematsu E, Ohno A, Ikejiri $\mathrm{H}$ and Kataoka $\mathrm{H}$ : A case of a pleomorphic hyalinizing angiectatic tumor of soft parts with intracytoplasmic hemosiderin pigment apparent upon fine-needle aspiration cytology. Diagn Cytopathol 43: 407-411, 2015.

40. Kane PM, Gaspar MP, Whiting BB and Culp RW: Pleomorphic hyalinizing angiectatic tumor arising in the hand: A Case Report. Hand (N Y) 11: NP20-NP23, 2016.

41. Onyemkpa $\mathrm{C}$ and Oyasiji T: Pleomorphic hyalinizing angiectatic tumor arising in the groin: A case report. Int J Surg Case Rep 28: 227-230, 2016.

This work is licensed under a Creative Commons Attribution-NonCommercial-NoDerivatives 4.0 International (CC BY-NC-ND 4.0) License. 